\title{
Guanidinium-tagged Organocatalysts for Direct Aldol Reactions
}

\author{
Jabbar Shah and Jürgen Liebscher \\ Institute of Chemistry, Humboldt-University Berlin, Brook-Taylor-Straße 2, 12489 Berlin, Germany \\ Reprint requests to Prof. J. Liebscher. E-mail: liebscher@chemie.hu-berlin.de
}

Z. Naturforsch. 2011, 66b, 88-94; received October 5, 2010

\begin{abstract}
Hexasubstituted guanidinium moieties have been used as ionic liquid tags for organocatalyts for the first time. Such conjugates were obtained in the $(S)$-proline and $(S)$-pyrrolidine-2-ylmethyl series by alkylation reactions of pentasubstituted guanidines. The resulting guanidinium-tagged organocatalysts were applied to asymmetric direct aldol reactions providing high stereoselectivities and yields and good recyclability, and thus performed better than $(S)$-proline itself.
\end{abstract}

Key words: Organocatalysis, Ionic Liquids, Guanidinium Salts, Proline, Asymmetric Synthesis, Aldol Reaction

\section{Introduction}

Organocatalysis is a rapidly developing field in asymmetric organic synthesis, and still new applications of hitherto not considered reactions are found. Thus the development of new catalysts is of high interest. From the point of view of Green Chemistry recycling of catalysts and solvents is an important issue. To meet this challenge the application of ionic liquids (IL's) was exploited [1 -8]. IL's possess very high boiling points and are almost non-volatile. Thus they can be used as reaction media from which volatile products can be removed by distillation, and the remaining IL's can be reused directly. As an alternative, products formed in a polar IL as reaction medium can be separated by extraction with unpolar solvents leaving behind the IL for reuse. However, if this methodology is used for organocatalyzed reactions, unwanted leaching of the organocatalyst can occur during extraction. To overcome such problems, so called IL-tagged organocatalysts have been developed [9]. Here, the organocatalyst unit is covalently linked to an IL motif. Thus this polar conjugate is less sensitive to leaching during extraction with organic solvents. IL-tagged organocatalysts can be used in IL media as well as in common organic solvents. So far, imidazolium salts were mainly used as IL tags of organocatalysts $[9,10]$, while guanidinium salts as another important class of IL's [11 - 18] were totally neglected. Recently, we used 1,2,3-triazolium tags [19-22], and we report here first examples of organocatalysts tagged by guanidinium salts and their application in direct asymmetric aldol reactions.
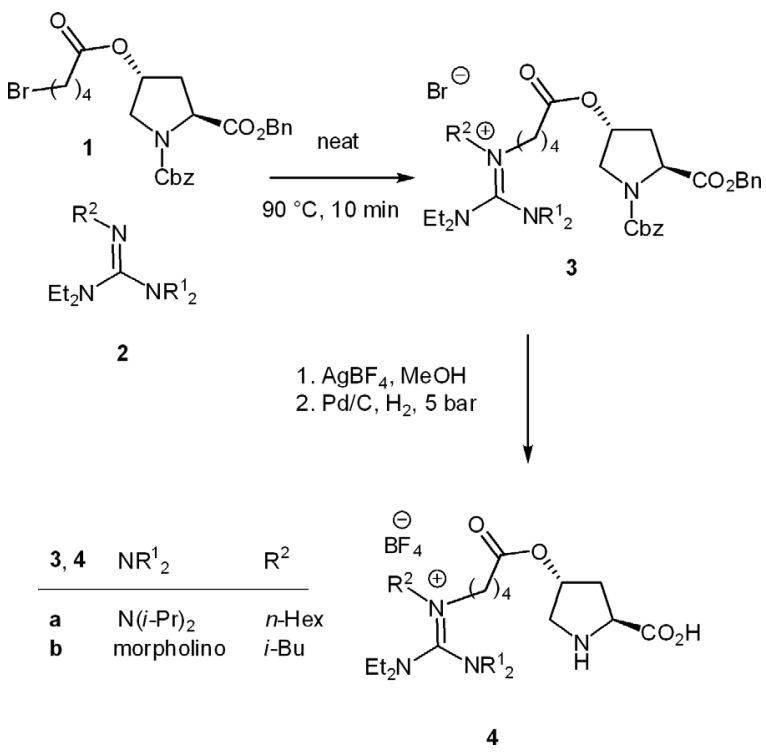

Scheme 1. Synthesis of guanidinium-tagged proline 4 .

\section{Results and Discussion}

The 4-hydroxy group of trans-(S)-4-hydroxyproline can be used as tethering site for IL tags resulting in conjugates of very good catalytic performance $[21,23-26]$. Accordingly, we used the 5bromopentanoylated $N$-Cbz- $(S)$-proline benzyl ester 1 and pentaalkyl-guanidines $\mathbf{2}$ to obtain the corresponding hexasubstituted guanidinium bromides $\mathbf{3}$ (Scheme 1).

After salt metathesis with Ag-tetrafluoroborate the proline moiety was deprotected by Pd-catalyzed hy- 


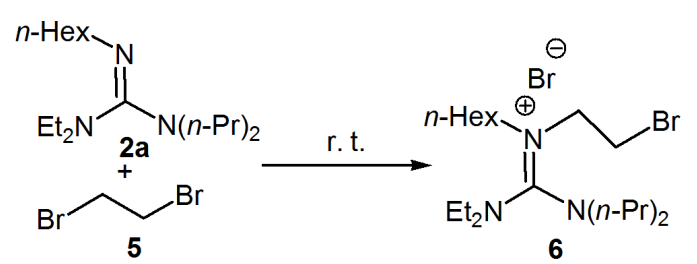

$\mathrm{HO}$

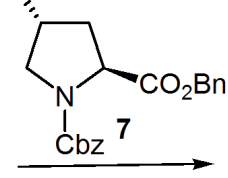

$\mathrm{NaH}, \mathrm{THF}$
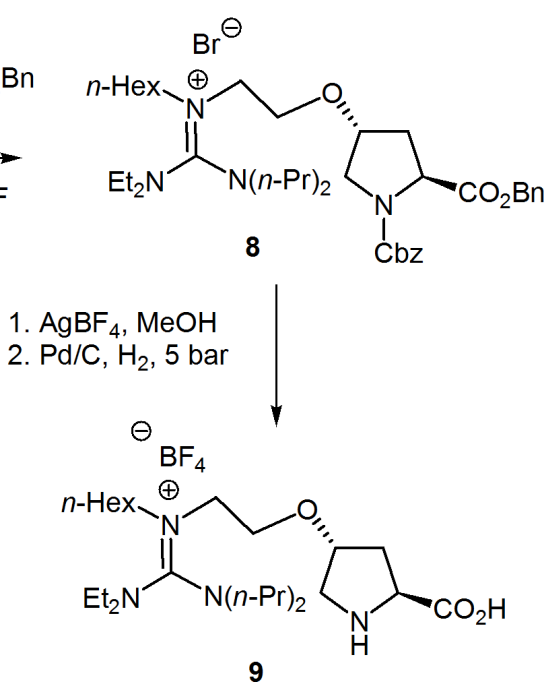

Scheme 2. Synthesis of the guanidinium-tagged proline $\mathbf{9}$.

drogenation providing the guanidinium-tagged prolines 4 in good yields. In another approach, the pentasubstituted guanidine 2a was alkylated with 1,2-dibromoethane, and the resulting guanidinium salt 6 served as alkylating reagent for the diprotected trans-(S)-4-hydroxyproline 7 giving the ether $\mathbf{8}$. Salt metathesis and hydrogenative deprotection provided the guanidinium-tagged proline $\mathbf{9}$ (Scheme 2).

In a different strategy, a chiral pyrrolidine $\mathbf{1 2}$ was targeted wherein the IL tag is connected to the methyl group in position 2 (Scheme 3 ). Thus $N$-Boc-protected prolinol tosylate $\mathbf{1 0}$ [27] was applied as alkylating reagent for the pentasubstituted guanidine 2a. Acidic deprotection of the resulting guanidinium salt $\mathbf{1 1}$ led to the free secondary amino group (formation of 12) necessary for enamine or iminium salt formation in organocatalysis.

With these IL-tagged organocatalysts $\mathbf{4 , 9}$ and $\mathbf{1 2}$ in hand, we applied them in direct aldol reactions of aromatic aldehydes 14 with cycloaliphatic and aliphatic ketones 13. The reactions were performed in an excess of ketone $\mathbf{1 3}$ as solvent with $20 \mathrm{~mol}-\%$ of the organocatalyst (Scheme 4).<smiles></smiles>

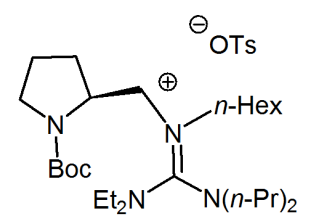

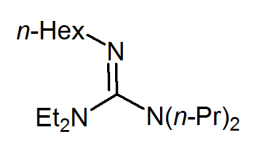

2a

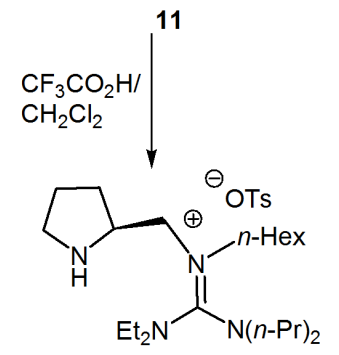

12

Scheme 3. Synthesis of the guanidinium-tagged methylpyrrolidine 12.<smiles>[R]CC([R])=O</smiles>

Scheme 4. Aldol reaction catalyzed by guanidinium-tagged organocatalysts 4,9 or 12 .

High yields (often above 90\%), diastereoselectivities (usually 95:5) and enantioselectivities (up to $98 \%$ ) were obtained in most cases (Table 1). While not much difference was found in the performance of the IL-tagged organocatalysts $4 a$ and $\mathbf{4 b}$, catalyst 9 showed less satisfactory results in the majority of cases. This fact demonstrates that the linker between the organocatalytic proline unit and the guanidinium tag has some effect on the catalytic properties. As far as the scope of reactants is concerned, better results were achieved with cyclohexanone than with cyclopentanone (compare Table 1, products 15a and 15f). Remarkably, also acetone could be transformed into the corresponding aldol $15 \mathrm{~g}$ in high yields and $e e$ up to $88 \%$ (Table 1). On the carbonyl side 14, aromatic aldehydes with electron withdrawing substituents perform better than those with electron donating groups (compare Table 1, products 15a and 15e). The guanidinium-tagged pyrrolidinemethylsubstituted guanidinium salt $\mathbf{1 2}$ was also applied to aldol reactions, but 15 was formed with no enantioselectivity. This phenomenon is probably caused by interaction of the secondary amino group with the guani- 
Table 1. Aldols 15 obtained with guanidinium-tagged organocatalysts 4 or 9.

\begin{tabular}{|c|c|c|c|c|c|c|c|c|c|c|c|c|c|c|}
\hline \multirow[b]{2}{*}{15} & \multirow[b]{2}{*}{$\mathrm{R}^{1} \mathrm{R}^{2}$} & \multirow[b]{2}{*}{$\mathrm{Ar}$} & \multicolumn{4}{|c|}{ Catalyst $\mathbf{4 a}$} & \multicolumn{4}{|c|}{ Catalyst $\mathbf{4 b}$} & \multicolumn{4}{|c|}{ Catalyst 9} \\
\hline & & & $\begin{array}{l}\mathrm{t} \\
\text { (h) }\end{array}$ & $\begin{array}{l}\text { Yield } \\
(\%)\end{array}$ & $\begin{array}{l}\text { antil } \\
\text { syn }^{\mathrm{a}}\end{array}$ & $\begin{array}{l}e e \\
(\%)^{\mathrm{a}}\end{array}$ & $\begin{array}{l}\mathrm{t} \\
\text { (h) }\end{array}$ & $\begin{array}{l}\text { Yield } \\
(\%)\end{array}$ & $\begin{array}{l}\text { antil } \\
\text { syn }^{\mathrm{a}}\end{array}$ & $\begin{array}{l}e e \\
(\%)^{\mathrm{a}}\end{array}$ & $\begin{array}{l}\mathrm{t} \\
\text { (h) }\end{array}$ & $\begin{array}{l}\text { Yield } \\
(\%)\end{array}$ & $\begin{array}{l}\text { anti / } \\
\text { syn }^{\mathrm{a}}\end{array}$ & $\begin{array}{l}e e \\
(\%)^{\mathrm{a}}\end{array}$ \\
\hline $\mathbf{a}$ & $\left(\mathrm{CH}_{2}\right)_{4}$ & $4-\mathrm{NO}_{2} \mathrm{Ph}$ & 28 & 94 & $97: 3$ & 98 & 31 & 99 & $97: 3$ & 92 & 25 & 92 & $97: 3$ & 94 \\
\hline b & $\left(\mathrm{CH}_{2}\right)_{4}$ & 4-Br-Ph & 29 & 89 & $95: 5$ & 72 & 24 & 90 & $95: 5$ & 64 & 36 & 87 & $95: 5$ & 76 \\
\hline c & $\left(\mathrm{CH}_{2}\right)_{4}$ & 4-Cl-Ph & 18 & 70 & $95: 5$ & 92 & 25 & 79 & $95: 5$ & 92 & 22 & 76 & $95: 5$ & 64 \\
\hline d & $\left(\mathrm{CH}_{2}\right)_{4}$ & 4-F-Ph & 12 & 90 & $90: 10$ & 94 & 17 & 95 & $90: 10$ & 88 & 27 & 86 & $90: 10$ & 70 \\
\hline $\mathbf{e}$ & $\left(\mathrm{CH}_{2}\right)_{4}$ & 4-MeOPh & 36 & 81 & $97: 3$ & 46 & 42 & 84 & $97: 3$ & 52 & 61 & 79 & $97: 3$ & 39 \\
\hline $\mathbf{f}$ & $\left(\mathrm{CH}_{2}\right)_{3}$ & $4-\mathrm{NO}_{2} \mathrm{Ph}$ & 44 & 87 & $67: 33$ & 86 & 41 & 91 & $67: 33$ & 81 & 50 & 81 & $67: 33$ & 74 \\
\hline g & $\mathrm{CH}_{3} \mathrm{H}$ & $4-\mathrm{NO}_{2} \mathrm{Ph}$ & 39 & 91 & - & 80 & 32 & 88 & - & 77 & 37 & 80 & - & 67 \\
\hline
\end{tabular}

a $e e$ of the major diastereomer, determined by HPLC on a chiral column (Chiralcel AD column ( $n$-hexane/ $i$-PrOH $90: 10)$, assignment of configuration by comparison with literature data: ref. [28]).

Table 2. Recycling experiments with IL-tagged catalyst 4a using Methods A, B, C.

\begin{tabular}{|c|c|c|c|c|c|c|c|c|c|}
\hline \multirow{3}{*}{$\mathrm{Cycle}^{\mathrm{a}}$} & \multicolumn{3}{|c|}{ Yield (\%)/ time $(\mathrm{h})^{\mathrm{a}}$} & \multicolumn{3}{|c|}{ anti/syn ${ }^{\mathrm{b}}$} & \multicolumn{3}{|c|}{$e e(\%)^{\mathrm{b}}$} \\
\hline & \multicolumn{3}{|c|}{ Method } & \multicolumn{3}{|c|}{ Method } & \multicolumn{3}{|c|}{ Method } \\
\hline & A & B & $\mathrm{C}$ & A & B & $\mathrm{C}$ & A & $\mathrm{B}$ & $\mathrm{C}$ \\
\hline 1 & $96 / 26$ & $97 / 21$ & $97 / 29$ & $99: 1$ & $97: 3$ & $98: 2$ & 98 & 98 & $\overline{99}$ \\
\hline 2 & $95 / 33$ & $96 / 28$ & $95 / 37$ & $98: 2$ & $97: 3$ & $98: 2$ & 98 & 95 & 98 \\
\hline 3 & $90 / 39$ & $93 / 35$ & $90 / 42$ & $95: 5$ & $96: 4$ & $95: 5$ & 94 & 85 & 77 \\
\hline 4 & $88 / 48$ & $92 / 45$ & $85 / 51$ & $95: 5$ & $96: 4$ & $94: 6$ & 79 & 72 & 62 \\
\hline
\end{tabular}
a Extraction of product with diethyl ether;
b ee of major diastereomer, determined
by HPLC on a chiral column (Chiralcel
AD column ( $n$-hexane/i-PrOH $90: 10)$;
assignment of configuration by compari-
son with literature data: ref. [28]).

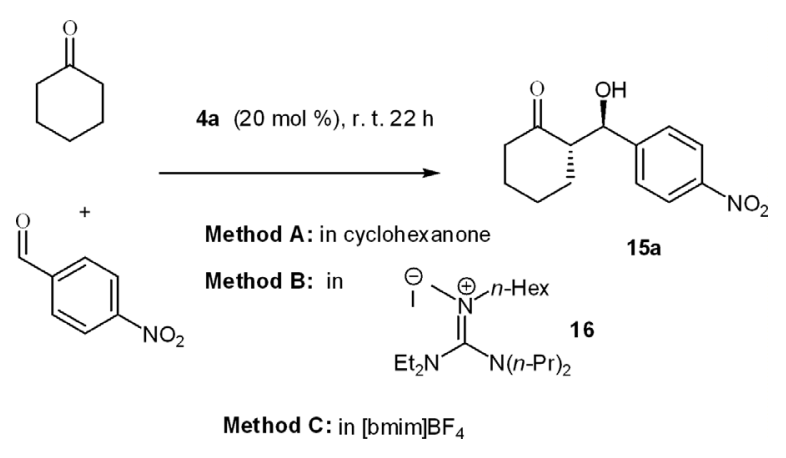

dinium $\mathrm{C}$ atom which reduces the nucleophilicity of the former and thus the enamine formation necessary for the aldol reaction is hampered.

At this point it can be resumed that guanidine tagging of proline results in effective catalysts for directed aldol reactions. In all cases results are much better than in analogous aldol reactions catalyzed just by proline [29].

As another issue, recycling of IL-tagged organocatalysts was investigated. The experiments were exerted with cyclohexanone and 4-nitrobenzaldehyde using three different methods A, B, C (Table 2). Method A was the same (excess cyclohexanone as solvent) as used above in Table 1. As an alternative, the reaction was performed with equimolar quantities of reactants in the guanidinium salt $\mathbf{1 6}$ as IL (Method B) or in the commercial IL 1-butyl-3-methylimidazolium tetrafluoroborate ([bmim $\left.] \mathrm{BF}_{4}\right)($ Method C). In each method, the product 15a was extracted with diethyl ether leav- ing behind 4a (Method A) or so-called working solutions of the IL with 4a (Methods B, C). New reactants were added to the remainder in order to perform the next run.

Table 2 reveals that $e e$ 's higher than $90 \%$ can be obtained up to the third run by method A. Methods B and $\mathrm{C}$ working in ionic liquids gave the same high $e e$ in the first run but showed a significant decrease after the second run. Diastereoselectivities were high throughout all experiments. The reduction of $e e$ 's after the third run observed in all cases was likely caused by gradual leaching of the IL-tagged organocatalyst during the extraction with diethyl ether.

\section{Conclusion}

In summary, ionic liquid tagging of organocatalysts by guanidinium salts was implemented for the first time. Such conjugates were obtained by alkylation of pentaalkyl-substituted guanidines with $(S)$-4hydroxyproline or an $(S)$-pyrrolidin-2-methyl derivative. Application in aldol reactions of ketones with aromatic aldehydes gave excellent yields and stereoselectivities which are significantly better than results obtained by $(S)$-proline, proving the usefulness of guanidinium salts as advantageous IL tags. As compared with IL-tagging of 4-hydroxyproline by 1,2,3-triazolium salts [19,21], the guanidinium-tagged organocatalysts reported here performed equally well but allowed improved recycling. 


\section{Experimental Section}

${ }^{1} \mathrm{H}$ NMR and ${ }^{13} \mathrm{C}$ NMR spectra in $\mathrm{CDCl}_{3}$ were recorded at 300 and $75 \mathrm{MHz}$, respectively, with a Bruker AC 300 instrument. Chemical shifts $\delta$ are given in ppm with TMS as internal standard. Silica gel $(0.04-0.063 \mathrm{~mm}$, Merck) was used for preparative column chromatography. Starting materials were purchased from commercial suppliers. Configurations of major products were elucidated by comparing their optical rotation with literature data [28]. Compounds 1, 7 and 10 [27] were synthesized according to literature procedures. Pentasubstituted guanidines $\mathbf{2}$ and the guanidinium iodide 16 were provided by Prof. W. Kantlehner, University of Stuttgart. All other materials were purchased from commercial suppliers.

\section{$N-((5-((3 R, 5 S)-5-C a r b o x y p y r r o l i d i n-3-y l o x y)-5-o x o p e n t y l)-$ $N-(($ diethylamino $)$ (dipropylamino)methylene)hexan-1- aminium tetrafluoroborate $(\mathbf{4 a})$}

A mixture of the proline derivative $1(1.10 \mathrm{~g}, 2.12 \mathrm{mmol})$ and 1,1-diethyl-2-hexyl-3,3-dipropylguanidine (2a) (0.72 g, $2.54 \mathrm{mmol}$ ) was heated to $90{ }^{\circ} \mathrm{C}$ for $10 \mathrm{~min}$, cooled to $20^{\circ} \mathrm{C}$ and washed with $\mathrm{Et}_{2} \mathrm{O}(6 \times 15 \mathrm{~mL})$. The residue was dissolved in $\mathrm{MeOH}(3 \mathrm{~mL})$, and $\mathrm{Et}_{2} \mathrm{O}(15 \mathrm{~mL})$ was gradually added to the stirred solution. The separated oil was put under vacuum ( 0.5 Torr) for $2 \mathrm{~h}$ to afford the bromide intermediate 3a. Yield: $1.29 \mathrm{~g}(76 \%)$; yellow, viscous oil. $R_{f}=0.21 .-{ }^{1} \mathrm{H} \mathrm{NMR}\left(300 \mathrm{MHz}, \mathrm{CDCl}_{3}\right): \delta=7.31-7.26$ $(\mathrm{m}, 10 \mathrm{H}, \mathrm{Ph}), 5.14-5.13\left(\mathrm{~m}, 2 \mathrm{H}, \mathrm{CH}_{2}-\mathrm{CO}_{2}-\mathrm{N}\right), 5.12(\mathrm{~m}$, $\left.2 \mathrm{H}, \mathrm{CH}_{2}-\mathrm{CO}_{2}-\mathrm{CH}\right), 5.03\left(\mathrm{~m}, 1 \mathrm{H}, \mathrm{CO}_{2}-\mathrm{CH}-\mathrm{C}\right), 4.42-4.38$ $\left(\mathrm{m}, 1 \mathrm{H}, \mathrm{N}-\mathrm{CH}-\mathrm{CO}_{2}\right), 4.22-4.19\left(\mathrm{~m}, 2 \mathrm{H}, \mathrm{N}^{+}-\mathrm{CH}_{2}-\left(\mathrm{CH}_{2}\right)_{3}-\right.$ $\mathrm{CO}), 4.11-4.07\left(\mathrm{~m}, 2 \mathrm{H}, \mathrm{N}^{+}{ }_{-} \mathrm{CH}_{2}-\left(\mathrm{CH}_{2}\right)_{4}-\mathrm{CH}_{3}\right), 3.88-3.76$ $\left(\mathrm{m}, 2 \mathrm{H}, \mathrm{N}-\mathrm{CH}_{2}-\mathrm{CO}\right), 2.67-2.61$ (dd, $4 \mathrm{H}, \mathrm{N}-\mathrm{CH}_{2}-\mathrm{CH}_{3}$ ), $2.56-2.52$ (t, $\left.4 \mathrm{H}, \mathrm{N}-\mathrm{CH}_{2}-\mathrm{CH}_{2}-\mathrm{CH}_{3}\right), 2.40-2.32(\mathrm{~m}, 1 \mathrm{H}$, $\left.\mathrm{CO}_{2}-\mathrm{CH}-\mathrm{CH}_{2}-\mathrm{CH}\right), 2.04-1.97\left(\mathrm{~m}, 1 \mathrm{H}, \mathrm{CO}_{2}-\mathrm{CH}-\mathrm{CH}_{2}-\mathrm{CH}\right)$, 2.37-2.25 (m, 2H, CO $\left.-\mathrm{CH}_{2}\right), 1.74-1.67\left(\mathrm{~m}, 2 \mathrm{H}, \mathrm{CO}_{2}\right.$ $\left.\mathrm{CH}_{2}-\mathrm{CH}_{2}\right), 1.85-1.78\left(\mathrm{~m}, 2 \mathrm{H}, \mathrm{CO}_{2}-\left(\mathrm{CH}_{2}\right)_{2}-\mathrm{CH}_{2}\right), 1.85-$ $1.78\left(\mathrm{~m}, 2 \mathrm{H}, \mathrm{N}^{+}-\mathrm{CH}_{2}-\mathrm{CH}_{2}-\left(\mathrm{CH}_{2}\right)_{2}-\mathrm{CO}\right), 1.74-1.67(\mathrm{~m}, 2 \mathrm{H}$, $\left.\mathrm{N}^{+}-\left(\mathrm{CH}_{2}\right)_{2}-\mathrm{CH}_{2}-\mathrm{CH}_{2}-\mathrm{CO}\right), 1.77-1.69\left(\mathrm{~m}, 2 \mathrm{H}, \mathrm{N}^{+}-\mathrm{CH}_{2}-\right.$ $\left.\mathrm{CH}_{2}-\left(\mathrm{CH}_{2}\right)_{3}-\right), 1.60-1.52\left(\mathrm{~m}, 2 \mathrm{H}, \mathrm{N}^{+}-\left(\mathrm{CH}_{2}\right)_{2}-\mathrm{CH}_{2}-\mathrm{CH}_{2}-\right.$ ), $1.29-1.20\left(\mathrm{~m}, 2 \mathrm{H}, \mathrm{N}^{+}-\left(\mathrm{CH}_{2}\right)_{3}-\mathrm{CH}_{2}-\right), 1.39-1.32(\mathrm{~m}$, $\left.2 \mathrm{H}, \mathrm{N}^{+}-\left(\mathrm{CH}_{2}\right)_{4}-\mathrm{CH}_{2}-\right), 1.44-1.35\left(\mathrm{~m}, 4 \mathrm{H}, \mathrm{CH}_{3}-\mathrm{CH}_{2}-\mathrm{CH}_{2}-\right.$ $\mathrm{N}), 0.96-0.94\left(\mathrm{~m}, 6 \mathrm{H}, \mathrm{CH}_{3}-\mathrm{CH}_{2}-\mathrm{N}\right), 0.81-0.78(\mathrm{~m}, 6 \mathrm{H}$, $\left.\mathrm{CH}_{3}-\left(\mathrm{CH}_{2}\right)_{2}-\mathrm{N}\right), 0.71\left(\mathrm{~m}, 3 \mathrm{H}, \mathrm{CH}_{3}-\left(\mathrm{CH}_{2}\right)_{5}-\mathrm{N}\right) .-{ }^{13} \mathrm{C} \mathrm{NMR}$ $\left(75 \mathrm{MHz}, \mathrm{CDCl}_{3}\right): \delta=171.1,168.6,154.6,136.3,135.7$, $127.0-128.4,73.2,67.4,67.1,62.8,53.2,53.1,51.4,47.9$, $43.3,36.1,33.4,31.2,28.6,28.1,26.9,22.2,21.6,19.3$, 13.7, 13.4, 8.1. - HRMS ((+)-ESI: $m / z=722.4969$ (calcd. 722.4971 for $\left.\mathrm{C}_{42} \mathrm{H}_{66} \mathrm{~N}_{4} \mathrm{O}_{6}{ }^{+},[\mathrm{M}+\mathrm{H}]^{+}\right)$.

For transformation into the corresponding tetrafluoroborate, the bromide $3 \mathbf{a}(0.5 \mathrm{~g}, 0.62 \mathrm{mmol})$ was dissolved in dry methanol $(15 \mathrm{~mL})$. In a second flask $\mathrm{AgBF}_{4}(0.12 \mathrm{~g}$, $0.62 \mathrm{mmol}$ ) was dissolved in dry methanol $(15 \mathrm{~mL})$ (to ex- clude light, the flask was wrapped in aluminum foil, or a brown flask was used). This $\mathrm{AgBF}_{4}$ solution was added dropwise to the solution of $\mathbf{3 a}$ until no more precipitate $(\mathrm{AgBr})$ was formed. After the precipitate had settled the supernatant clear solution was separated, dried and evaporated giving a quantitative yield of the tetrafluoroborate as a yellow oil. The NMR spectra are identical to those of 3a. For deprotection, to a solution of the tetrafluoroborate $(1 \mathrm{~g}, 1.23 \mathrm{mmol})$ in dry methanol $(15 \mathrm{~mL}) \mathrm{Pd} / \mathrm{C}(80 \mathrm{mg})$ was added, and the mixture was pressurized under $\mathrm{H}_{2}$ at 5 bar. After stirring over night the $\mathrm{Pd} / \mathrm{C}$ was filtered off, and the filtrate was concentrated under vacuum giving the final product 4a. Yield: $611 \mathrm{mg}(85 \%)$; yellow oil. - ${ }^{1} \mathrm{H}$ NMR $\left(300 \mathrm{MHz}, \mathrm{CDCl}_{3}\right)$ : $\delta=5.22\left(\mathrm{~m}, 1 \mathrm{H}, \mathrm{CH}_{2}-\mathrm{CO}_{2}-\mathrm{CH}\right), 4.97\left(\mathrm{~m}, 2 \mathrm{H}, \mathrm{N}^{+}-\mathrm{CH}_{2}-\right.$ $\left.\left(\mathrm{CH}_{2}\right)_{4}-\mathrm{CH}_{3}\right), 4.22\left(\mathrm{~m}, 1 \mathrm{H}, \mathrm{N}-\mathrm{CH}-\mathrm{CO}_{2}\right), 3.24\left(\mathrm{~m}, 2 \mathrm{H}, \mathrm{N}^{+}\right.$. $\left.\mathrm{CH}_{2}-\left(\mathrm{CH}_{2}\right)_{3}-\mathrm{CO}\right), 3.00\left(\mathrm{~m}, 2 \mathrm{H}, \mathrm{N}-\mathrm{CH}_{2}-\mathrm{CH}-\mathrm{O}\right), 2.88(\mathrm{~m}, 1 \mathrm{H}$, $\left.\mathrm{CO}_{2}-\mathrm{CH}-\mathrm{CH}_{2}-\mathrm{CH}\right), 2.52\left(\mathrm{~m}, 4 \mathrm{H}, \mathrm{N}-\mathrm{CH}_{2}-\mathrm{CH}_{3}\right), 2.45(\mathrm{~m}, 1 \mathrm{H}$, $\left.\mathrm{CO}_{2}-\mathrm{CH}-\mathrm{CH}_{2}-\mathrm{CH}\right), 2.29$ (t, $\left.4 \mathrm{H}, \mathrm{N}-\mathrm{CH}_{2}-\mathrm{CH}_{2}-\mathrm{CH}_{3}\right), 1.61$ $\left(\mathrm{m}, 2 \mathrm{H}, \mathrm{CO}_{2}-\mathrm{CH}_{2}\right), 1.42\left(\mathrm{~m}, 2 \mathrm{H}, \mathrm{N}^{+}-\mathrm{CH}_{2}-\mathrm{CH}_{2}-\left(\mathrm{CH}_{2}\right)_{2}-\right.$ $\mathrm{CO}), 1.25\left(\mathrm{~m}, 2 \mathrm{H}, \mathrm{N}^{+}-\mathrm{CH}_{2}-\mathrm{CH}_{2}-\left(\mathrm{CH}_{2}\right)_{3}-\right), 1.17(\mathrm{~m}, 2 \mathrm{H}$, $\left.\mathrm{N}^{+}-\left(\mathrm{CH}_{2}\right)_{2}-\mathrm{CH}_{2}-\mathrm{CH}_{2}-\mathrm{CO}\right), 1.11\left(\mathrm{~m}, 2 \mathrm{H}, \mathrm{N}^{+}-\left(\mathrm{CH}_{2}\right)_{2}-\mathrm{CH}_{2}-\right.$ $\left.\mathrm{CH}_{2}-\right), 1.06\left(\mathrm{~m}, 4 \mathrm{H}, \mathrm{CH}_{3}-\mathrm{CH}_{2}-\mathrm{CH}_{2}-\mathrm{N}\right), 1.01\left(\mathrm{~m}, 2 \mathrm{H}, \mathrm{N}^{+}\right.$$\left.\left(\mathrm{CH}_{2}\right)_{4}-\mathrm{CH}_{2}-\right), 0.98\left(\mathrm{~m}, 2 \mathrm{H}, \mathrm{N}^{+}-\left(\mathrm{CH}_{2}\right)_{3}-\mathrm{CH}_{2}-\right), 0.98-0.94$ (m, 6H, $\left.\mathrm{CH}_{3}-\mathrm{CH}_{2}-\mathrm{N}\right), 0.81\left(\mathrm{~m}, 6 \mathrm{H}, \mathrm{CH}_{3}-\left(\mathrm{CH}_{2}\right)_{2}-\mathrm{N}\right), 0.76$ $\left(\mathrm{m}, 3 \mathrm{H}, \mathrm{CH}_{3}-\left(\mathrm{CH}_{2}\right)_{5}-\mathrm{N}\right) .-{ }^{13} \mathrm{C}$ NMR $\left(75 \mathrm{MHz}, \mathrm{CDCl}_{3}\right)$ : $\delta=172.9,163.8,135.7,73.0,60.9,51.1,50.9,49.2,43.8$, $34.8,33.5,32.8,29.0,28.7,26.9,22.0,21.4,20.5,13.7$, 10.5. - HRMS ((+)-ESI: $m / z=498.4133$ (calcd. 498.4134 for $\left.\mathrm{C}_{27} \mathrm{H}_{54} \mathrm{~N}_{4} \mathrm{O}_{4}{ }^{+},[\mathrm{M}+\mathrm{H}]^{+}\right)$.

\section{5-((3R,5S)-5-Carboxypyrrolidin-3-yloxy)- $N-(($ diethyl- amino)(morpholino)methylene)- $N$-isobutyl-5-oxopentan-1- aminium tetrafluoroborate $(\mathbf{4 b})$}

A mixture of the 4-( $\omega$-bromovaleroyloxy)-proline $\mathbf{1}$ $(1.10 \mathrm{~g}, 2.12 \mathrm{mmol})$ and the guanidine $\mathbf{2 b}(0.61 \mathrm{~g}$, $2.54 \mathrm{mmol})$ was heated at $90{ }^{\circ} \mathrm{C}$ for $10 \mathrm{~min}$, cooled to $20{ }^{\circ} \mathrm{C}$ and washed with $\mathrm{Et}_{2} \mathrm{O}(6 \times 15 \mathrm{~mL})$. The residue was dissolved in $\mathrm{MeOH}(3 \mathrm{~mL})$, and $\mathrm{Et}_{2} \mathrm{O}(15 \mathrm{~mL})$ was gradually added to the stirred solution. The separated oil was put under vacuum (0.5 Torr) for $2 \mathrm{~h}$ to afford the guanidinium bromide $3 \mathrm{~b}$. Yield: $1.41 \mathrm{~g}(88 \%)$; yellow viscous oil. $-{ }^{1} \mathrm{H}$ NMR (300 MHz, $\left.\mathrm{CDCl}_{3}\right): \delta=7.34-7.15(\mathrm{~m}$, $10 \mathrm{H}, \mathrm{Ar}), 5.21-5.06\left(\mathrm{~m}, 4 \mathrm{H}, \mathrm{CH}_{2}-\mathrm{CO}_{2}-\mathrm{CH}\right), 4.98-4.92(\mathrm{~m}$, $\left.1 \mathrm{H}, \mathrm{CO}_{2}-\mathrm{CH}-\mathrm{C}\right), 4.51-4.31\left(\mathrm{~m}, 1 \mathrm{H}, \mathrm{N}-\mathrm{CH}-\mathrm{CO}_{2}\right), 3.91-$ $3.81\left(\mathrm{~m}, 2 \mathrm{H}, \mathrm{N}^{+}-\mathrm{CH}_{2}-\left(\mathrm{CH}_{2}\right)_{3}-\mathrm{CO}\right), 3.78-3.64(\mathrm{~m}, 2 \mathrm{H}$, $\left.\mathrm{N}^{+}-\mathrm{CH}_{2}-\mathrm{CH}-\left(\mathrm{CH}_{3}\right)_{2}\right), 3.60-3.54\left(\mathrm{~m}, 2 \mathrm{H}, \mathrm{N}-\mathrm{CH}_{2}-\mathrm{CH}-\mathrm{O}\right)$, $3.49-3.44\left(\mathrm{~m}, 4 \mathrm{H}, \mathrm{N}-\mathrm{CH}_{2}-\mathrm{CH}_{2}-\mathrm{O}\right), 3.42-3.36(\mathrm{dd}, 4 \mathrm{H}$, $\left.\mathrm{N}-\mathrm{CH}_{2}-\mathrm{CH}_{3}\right), 3.23-3.19\left(\mathrm{~m}, 4 \mathrm{H}, \mathrm{N}-\mathrm{CH}_{2}-\mathrm{CH}_{2}-\mathrm{O}\right), 2.96-$ $2.57\left(\mathrm{~m}, 1 \mathrm{H}, \mathrm{CO}_{2}-\mathrm{CH}-\mathrm{CH}_{2}-\mathrm{CH}\right), 2.43\left(\mathrm{~m}, 2 \mathrm{H}, \mathrm{CO}_{2}-\mathrm{CH}_{2}-\right.$ $\left.\mathrm{CH}_{2}\right), 2.04-1.97\left(\mathrm{~m}, 1 \mathrm{H}, \mathrm{CO}_{2}-\mathrm{CH}-\mathrm{CH}_{2}-\mathrm{CH}\right), 2.18-1.88$ $\left(\mathrm{m}, 2 \mathrm{H}, \mathrm{N}^{+}-\mathrm{CH}_{2}-\mathrm{CH}_{2}-\left(\mathrm{CH}_{2}\right)_{2}-\mathrm{CO}\right), 1.65\left(\mathrm{~m}, 2 \mathrm{H}, \mathrm{N}^{+}-\right.$ $\left.\left(\mathrm{CH}_{2}\right)_{2}-\mathrm{CH}_{2}-\mathrm{CH}_{2}-\mathrm{CO}\right), \mathrm{l} .60\left(\mathrm{~m}, 1 \mathrm{H}, \mathrm{N}^{+}-\mathrm{CH}_{2}-\mathrm{CH}-\left(\mathrm{CH}_{3}\right)_{2}\right)$, 
$1.27-1.16\left(\mathrm{~m}, 6 \mathrm{H}, \mathrm{N}^{+}-\mathrm{CH}_{2}-\mathrm{CH}-\left(\mathrm{CH}_{3}\right)_{2}\right), 0.92-0.79(\mathrm{~m}$, $\left.6 \mathrm{H}, \mathrm{CH}_{3}-\mathrm{CH}_{2}-\mathrm{N}\right) .-{ }^{13} \mathrm{C} \mathrm{NMR}\left(75 \mathrm{MHz}, \mathrm{CDCl}_{3}\right): \delta=$ $172.7,172.01,163.2,154.6,154.1,136.1,135.3,128.5-$ $127.1,71.8,67.2,66.7,59.8,57.6,52.6,50.9,49.9,44.8$, 43.6, 36.4, 35.4, 26.8, 26.4, 20.5, 20.3, 20.2, 13.2, 13.0. HRMS ((+)-ESI: $m / z=680.4136$ (calcd. 680.4138 for $\left.\mathrm{C}_{38} \mathrm{H}_{56} \mathrm{~N}_{4} \mathrm{O}_{7},[\mathrm{M}+\mathrm{H}]^{+}\right)$.

The guanidinium bromide $\mathbf{3 b}$ was transformed into the corresponding tetrafluoroborate and finally deprotected in the same way as described for $\mathbf{4 a}$ giving the final product $\mathbf{4 b}$. Yield: $585 \mathrm{mg}(83 \%)$; yellow oil. $-{ }^{1} \mathrm{H}$ NMR $(300 \mathrm{MHz}$, $\left.\mathrm{CDCl}_{3}\right): \delta=5.28\left(\mathrm{~m}, 1 \mathrm{H}, \mathrm{CH}_{2}-\mathrm{CO}_{2}-\mathrm{CH}-\mathrm{C}\right), 4.01(\mathrm{~m}, 1 \mathrm{H}, \mathrm{N}-$ $\left.\mathrm{CH}-\mathrm{CO}_{2}\right), 3.98\left(\mathrm{~m}, 2 \mathrm{H}, \mathrm{N}^{+}-\mathrm{CH}_{2}-\left(\mathrm{CH}_{2}\right)_{3}-\mathrm{CO}\right), 3.74(\mathrm{~m}, 2 \mathrm{H}$, $\left.\mathrm{N}^{+}-\mathrm{CH}_{2}-\mathrm{CH}-\left(\mathrm{CH}_{3}\right)_{2}\right), 3.38$ (m, 2H, N-CH $\left.-\mathrm{CH}-\mathrm{O}\right), 3.20$ (m, $\left.4 \mathrm{H}, \mathrm{N}-\mathrm{CH}_{2}-\mathrm{CH}_{2}-\mathrm{O}\right), 2.72-2.30$ (dd, $4 \mathrm{H}, \mathrm{N}-\mathrm{CH}_{2}-\mathrm{CH}_{3}$ ), $2.40-2.31\left(\mathrm{~m}, 4 \mathrm{H}, \mathrm{N}-\mathrm{CH}_{2}-\mathrm{CH}_{2}-\mathrm{O}\right), 1.76-1.43(\mathrm{~m}, 1 \mathrm{H}$, $\left.\mathrm{CO}_{2}-\mathrm{CH}-\mathrm{CH}_{2}-\mathrm{CH}\right), 1.74-1.67\left(\mathrm{~m}, 2 \mathrm{H}, \mathrm{N}^{+}-\left(\mathrm{CH}_{2}\right)_{2}-\mathrm{CH}_{2}-\right.$ $\left.\mathrm{CH}_{2}-\mathrm{CO}\right), 1.62\left(\mathrm{~m}, 2 \mathrm{H}, \mathrm{CO}_{2}-\mathrm{CH}_{2}\right), 1.56\left(\mathrm{~m}, 2 \mathrm{H}, \mathrm{N}^{+}-\mathrm{CH}_{2}-\right.$ $\left.\mathrm{CH}_{2}-\left(\mathrm{CH}_{2}\right)_{2}-\mathrm{CO}\right), 1.22\left(\mathrm{~m}, 1 \mathrm{H}, \mathrm{N}^{+}-\mathrm{CH}_{2}-\mathrm{CH}-\left(\mathrm{CH}_{3}\right)_{2}\right), 0.87$ $\left(\mathrm{m}, 6 \mathrm{H}, \mathrm{N}^{+}-\mathrm{CH}_{2}-\mathrm{CH}-\left(\mathrm{CH}_{3}\right)_{2}\right), 0.76\left(\mathrm{~m}, 6 \mathrm{H}, \mathrm{CH}_{3}-\mathrm{CH}_{2}-\mathrm{N}\right)$. ${ }^{13} \mathrm{C} \mathrm{NMR}\left(75 \mathrm{MHz}, \mathrm{CDCl}_{3}\right): \delta=175.1,171.6,163.1,133.5$, 73.2, 65.8, 65.5, 65.4, 64.5, 57.5, 56.4, 49.8, 48.9, 43.8, 36.1, 33.5, 26,3, 25.9, 21.4, 20.2, 12.7. - HRMS: $((+)-E S I: ~ m / z=$ 456.3300 (calcd. 456.3301 for $\mathrm{C}_{23} \mathrm{H}_{44} \mathrm{~N}_{4} \mathrm{O}_{5},[\mathrm{M}+\mathrm{H}]^{+}$).

\section{$N$-(2-Bromoethyl)- $N$-((diethylamino)(dipropylamino)- methylene)hexane-1-aminium bromide (6)}

1,1-Diethyl-2-hexyl-3,3-dipropylguanidine (2a) (1.00 g, $3.7 \mathrm{mmol})$ was mixed with 1,2-dibromoethane (1.09 $\mathrm{g}$, $5.5 \mathrm{mmol}$ ). The reaction mixture was stirred at $\mathrm{r}$. t. over night. The resulting sticky matarial was washed with diethyl ether $(3 \times 25 \mathrm{~mL})$. After keeping under vacuum for $1 \mathrm{~h}$ product 6 was obtained as a colorless oil; yield $1.3 \mathrm{~g}(64 \%) .-{ }^{1} \mathrm{H}$ NMR $\left(300 \mathrm{MHz} \mathrm{CDCl}_{3}\right): \delta=4.09-3.91\left(\mathrm{~m}, 2 \mathrm{H}, \mathrm{N}^{+}-\mathrm{CH}_{2}-\right.$ $\mathrm{CH}_{2}-\mathrm{O}$ ), $3.79-3.58\left(\mathrm{~m}, 2 \mathrm{H}, \mathrm{N}^{+}-\mathrm{CH}_{2}-\mathrm{CH}_{2}-\mathrm{O}\right), 3.41-2.91$ $\left(\mathrm{m}, 10 \mathrm{H}, \mathrm{N}\left(\mathrm{CH}_{2}-\mathrm{CH}_{2}-\mathrm{CH}_{3}\right)_{2}, \mathrm{~N}\left(\mathrm{CH}_{2}-\left(\mathrm{CH}_{2}\right)_{4} \mathrm{CH}_{3}\right), \mathrm{N}\left(\mathrm{CH}_{2}-\right.\right.$ $\left.\left.\mathrm{CH}_{3}\right)_{2}\right), 1.79-1.43\left(\mathrm{~m}, 6 \mathrm{H}, \mathrm{N}\left(\mathrm{CH}_{2}-\mathrm{CH}_{3}\right)_{2}\right), 1.22-1.27$ $\left.\left(\mathrm{m}, 12 \mathrm{H}, \mathrm{N}-\left(\mathrm{CH}_{2}\right)-\left(\mathrm{CH}_{2}\right)_{4}-\mathrm{CH}_{3}\right), \mathrm{N}-\left(\left(\mathrm{CH}_{2}\right)-\left(\mathrm{CH}_{2}\right)-\mathrm{CH}_{3}\right)_{2}\right)$, $0.86-0.96\left(\mathrm{~m}, 9 \mathrm{H}, \mathrm{N}\left(\mathrm{CH}_{2}-\mathrm{CH}_{2}-\mathrm{CH}_{3}\right)_{2}, \mathrm{~N}-\left(\mathrm{CH}_{2}\right)_{5}-\mathrm{CH}_{3}\right)$. ${ }^{13} \mathrm{C} \mathrm{NMR}\left(75 \mathrm{MHz} \mathrm{CDCl}_{3}\right): \delta=163.78,53.24,53.15,51.69$, 51.56, 51.07, 31.26, 27.40, 27.22, 26.46, 22.48, 20.97, 13.93, $13.55,13.26,12.97,11.37$.

$N-(2-((3 R, 5 S)-5$-Carboxypyrrolidin-3-yloxy)ethyl)- $N$ ((diethylamino)(dipropylamino)methylene)hexan-1-aminium tetrafluoroborate (9)

A solution of the diprotected 4-hydroxyproline 7 (3.2 g, $9 \mathrm{mmol})$ in dry DMF $(25 \mathrm{~mL})$ was added to a suspension of sodium hydride $(0.3 \mathrm{~g}, 13 \mathrm{mmol})$ in dry DMF $(25 \mathrm{~mL})$ at $-20{ }^{\circ} \mathrm{C}$ under argon. The reaction mixture was stirred for $20 \mathrm{~min}$. $N$-(2-Bromoethyl)- $N$-((diethylamino)(dipropylamino)methylene)hexan-1-aminium bromide (6) (6.12 g,
$13 \mathrm{mmol}$ ) was added, and the reaction mixture was stirred at r. t. over night. The reaction mixture was extracted with diethyl ether $(3 \times 50 \mathrm{~mL})$, giving a quantitative yield of 8 . ${ }^{1} \mathrm{H} \mathrm{NMR}\left(300 \mathrm{MHz}, \mathrm{CDCl}_{3}\right): \delta=7.11-6.87$ (m, 10H, Ar), 5.10-5.02 (m, 4H, Ph- $\left.\mathrm{CH}_{2}-\mathrm{CO}_{2}\right), 4.95-4.77\left(\mathrm{~m}, 1 \mathrm{H}, \mathrm{CO}_{2}\right.$ $\mathrm{CH}-\mathrm{C}), 4.16-4.02\left(\mathrm{~m}, 1 \mathrm{H}, \mathrm{N}-\mathrm{CH}-\mathrm{CO}_{2}\right), 3.83-3.76(\mathrm{~m}, 2 \mathrm{H}$, $\left.\mathrm{N}^{+}-\mathrm{CH}_{2}-\mathrm{CH}_{2}-\mathrm{O}\right), 3.61-3.47\left(\mathrm{~m}, 1 \mathrm{H}, \mathrm{O}-\mathrm{CH}-\mathrm{CH}_{2}\right), 4.11-$ $4.07\left(\mathrm{~m}, 2 \mathrm{H}, \mathrm{N}^{+}-\mathrm{CH}_{2}-\left(\mathrm{CH}_{2}\right)_{4}-\mathrm{CH}_{3}\right), 3.92-3.80(\mathrm{~m}, 2 \mathrm{H}$, $\left.\mathrm{N}^{+}-\mathrm{CH}_{2}-\mathrm{CH}_{2}-\mathrm{O}\right), 3.71-3.52\left(\mathrm{~m}, 2 \mathrm{H}, \mathrm{N}-\mathrm{CH}_{2}-\mathrm{CO}\right), 2.94-$ 2.80 (dd, $4 \mathrm{H}, \mathrm{N}-\mathrm{CH}_{2}-\mathrm{CH}_{3}$ ), $2.56-2.52$ (t, $4 \mathrm{H}, \mathrm{N}-\mathrm{CH}_{2}-\mathrm{CH}_{2}-$ $\left.\mathrm{CH}_{3}\right), 2.40-2.32\left(\mathrm{~m}, 1 \mathrm{H}, \mathrm{CO}_{2}-\mathrm{CH}-\mathrm{CH}_{2}-\mathrm{CH}\right), 1.82-1.64$ $\left(\mathrm{m}, 1 \mathrm{H}, \mathrm{CO}_{2}-\mathrm{CH}-\mathrm{CH}_{2}-\mathrm{CH}\right), 1.77-1.69\left(\mathrm{~m}, 2 \mathrm{H}, \mathrm{N}^{+}-\mathrm{CH}_{2}-\right.$ $\left.\mathrm{CH}_{2}-\left(\mathrm{CH}_{2}\right)_{3}-\right), 1.60-1.52\left(\mathrm{~m}, 2 \mathrm{H}, \mathrm{N}^{+}-\left(\mathrm{CH}_{2}\right)_{2}-\mathrm{CH}_{2}-\mathrm{CH}_{2}-\right)$, $1.29-1.20\left(\mathrm{~m}, 2 \mathrm{H}, \mathrm{N}^{+}-\left(\mathrm{CH}_{2}\right)_{3}-\mathrm{CH}_{2}-\right), 1.18-1.14(\mathrm{~m}, 2 \mathrm{H}$, $\left.\mathrm{N}^{+}-\left(\mathrm{CH}_{2}\right)_{4}-\mathrm{CH}_{2}-\right), 1.01-0.91\left(\mathrm{~m}, 4 \mathrm{H}, \mathrm{CH}_{3}-\mathrm{CH}_{2}-\mathrm{CH}_{2}-\mathrm{N}\right)$, $0.78-0.72\left(\mathrm{~m}, 6 \mathrm{H}, \mathrm{CH}_{3}-\mathrm{CH}_{2}-\mathrm{N}\right), 0.61-0.56\left(\mathrm{~m}, 6 \mathrm{H}, \mathrm{CH}_{3}-\right.$ $\left.\left(\mathrm{CH}_{2}\right)_{2}-\mathrm{N}\right), 0.55-0.52\left(\mathrm{~m}, 3 \mathrm{H}, \mathrm{CH}_{3}-\left(\mathrm{CH}_{2}\right)_{5}-\mathrm{N}\right) .-{ }^{13} \mathrm{C} \mathrm{NMR}$ $\left(75 \mathrm{MHz}, \mathrm{CDCl}_{3}\right): \delta=170.4,159.7,159.1,154.1,142.6$, 127.6-126.1, 77.1, 63.3, 59.8, 57.6, 50.6, 49.4, 42.3, 41.9, $31.1,29.4,22.2,21.2,21.1,20.5,13.8,13.0,12.6,11,1$. HRMS: $((+)$-ESI: $m / z=666.4708$ (calcd. 666.4709 for $\left.\mathrm{C}_{39} \mathrm{H}_{62} \mathrm{~N}_{4} \mathrm{O}_{5}{ }^{+},[\mathrm{M}+\mathrm{H}]^{+}\right)$.

In a $50 \mathrm{~mL}$ dry round bottom flask the bromide $8(0.5 \mathrm{~g}$, $0.67 \mathrm{mmol}$ ) was dissolved in $15 \mathrm{~mL}$ of dry methanol $(15 \mathrm{~mL})$. $\mathrm{AgBF}_{4}(0.13 \mathrm{~g}, 0.67 \mathrm{mmol})$ was dissolved in dry methanol $(15 \mathrm{~mL})$ under the exclusion of light. This $\mathrm{AgBF}_{4}$ solution was added dropwise to the solution of $\mathbf{8}$ until no more precipitate $(\mathrm{AgBr})$ was formed. After the precipitate had settled down the supernatant clear solution was separated, dried and evaporated to give a quantitative yield of the corresponding guanidinium tetrafluoroborate as a yellow oil. It was dissolved in dry methanol $(1.00 \mathrm{~g}, 1.3 \mathrm{mmol}$ in $15 \mathrm{~mL})$, and $\mathrm{Pd} / \mathrm{C}(80 \mathrm{mg})$ was added. The mixture was pressurized under $\mathrm{H}_{2}$ at 5 bar. After stirring over night the $\mathrm{Pd} / \mathrm{C}$ was filtered off, and the filtrate was concentrated under vacuum giving the unprotected tetrafluoroborate $9(0.62 \mathrm{~g}$, $91 \%)$ as a yellow oil. $-{ }^{1} \mathrm{H}$ NMR $\left(300 \mathrm{MHz}, \mathrm{CDCl}_{3}\right)$ : $\delta=4.25\left(\mathrm{~m}, 2 \mathrm{H}, \mathrm{N}^{+}-\mathrm{CH}_{2}-\mathrm{CH}_{2}-\mathrm{O}\right), 4.08(\mathrm{~m}, 1 \mathrm{H}, \mathrm{N}-\mathrm{CH}-$ $\left.\mathrm{CO}_{2}\right), 3.95-3.88\left(\mathrm{~m}, 1 \mathrm{H}, \mathrm{O}-\mathrm{CH}-\mathrm{CH}_{2}\right), 4.11-4.07(\mathrm{~m}, 2 \mathrm{H}$, $\left.\mathrm{N}^{+}-\mathrm{CH}_{2}-\left(\mathrm{CH}_{2}\right)_{4}-\mathrm{CH}_{3}\right), 3.92-3.78\left(\mathrm{~m}, 2 \mathrm{H}, \mathrm{N}^{+}-\mathrm{CH}_{2}-\mathrm{CH}_{2}-\right.$ O), $3.91-3.76\left(\mathrm{~m}, 2 \mathrm{H}, \mathrm{N}-\mathrm{CH}_{2}-\mathrm{CO}\right), 2.67-2.61(\mathrm{dd}, 4 \mathrm{H}$, $\left.\mathrm{N}-\mathrm{CH}_{2}-\mathrm{CH}_{3}\right), 2.56-2.52\left(\mathrm{t}, 4 \mathrm{H}, \mathrm{N}-\mathrm{CH}_{2}-\mathrm{CH}_{2}-\mathrm{CH}_{3}\right), 2.40-$ $2.32\left(\mathrm{~m}, 1 \mathrm{H}, \mathrm{CO}_{2}-\mathrm{CH}-\mathrm{CH}_{2}-\mathrm{CH}\right), 2.04-1.97\left(\mathrm{~m}, 1 \mathrm{H}, \mathrm{CO}_{2}-\right.$ $\left.\mathrm{CH}-\mathrm{CH}_{2}-\mathrm{CH}\right), 1.87-1.69\left(\mathrm{~m}, 2 \mathrm{H}, \mathrm{N}^{+}-\mathrm{CH}_{2}-\mathrm{CH}_{2}-\left(\mathrm{CH}_{2}\right)_{3}-\right.$ ), $1.60-1.52\left(\mathrm{~m}, 2 \mathrm{H}, \mathrm{N}^{+}-\left(\mathrm{CH}_{2}\right)_{2}-\mathrm{CH}_{2}-\mathrm{CH}_{2}-\right), 1.25-1.19$ $\left(\mathrm{m}, 2 \mathrm{H}, \mathrm{N}^{+}-\left(\mathrm{CH}_{2}\right)_{3}-\mathrm{CH}_{2}-\right), 1.39-1.29\left(\mathrm{~m}, 2 \mathrm{H}, \mathrm{N}^{+}-\left(\mathrm{CH}_{2}\right)_{4}^{-}\right.$ $\left.\mathrm{CH}_{2}-\right), 1.39-1.35\left(\mathrm{~m}, 4 \mathrm{H}, \mathrm{CH}_{3}-\mathrm{CH}_{2}-\mathrm{CH}_{2}-\mathrm{N}\right), 0.96-0.94$ (m, 6H, $\left.\mathrm{CH}_{3}-\mathrm{CH}_{2}-\mathrm{N}\right), 0.81-0.78\left(\mathrm{~m}, 6 \mathrm{H}, \mathrm{CH}_{3}-\left(\mathrm{CH}_{2}\right)_{2}-\mathrm{N}\right)$, 0.66-.59 (m, 3H, $\left.\mathrm{CH}_{3}-\left(\mathrm{CH}_{2}\right)_{5}-\mathrm{N}\right) .-{ }^{13} \mathrm{C} \mathrm{NMR}(75 \mathrm{MHz}$, $\left.\mathrm{CDCl}_{3}\right): \delta=172.1,148.6,77.2,61.4,60.7,56.5,52.9$, 51.4, 45.5, 43.3, 32.5, 31.2, 28.6, 26.9, 22.2, 19.3, 13.7, 8.1. - HRMS: ((+)-ESI: $m / z=442.3870$ (calcd. 442.3872 for $\mathrm{C}_{24} \mathrm{H}_{50} \mathrm{~N}_{4} \mathrm{O}_{3}{ }^{+},[\mathrm{M}+\mathrm{H}]^{+}$) 
(S)-N-((Diethylamino)(hexyl(pyrrolidin-2-ylmethyl)amino)methylene)- $N$-propylpropan-1-aminium 4-methylbenzenesulfonate (12)

A mixture of $(S)-N$-Boc- $O$-tosylprolinol $\mathbf{1 0}(1.00 \mathrm{~g}$, $2.81 \mathrm{mmol}$ ) and the pentasubstituted guanidine $2 \mathrm{a}$ (1.2 eq., $955 \mathrm{mg}, 3.37 \mathrm{mmol}$ ) was heated to $90{ }^{\circ} \mathrm{C}$ for $1 \mathrm{~h}$, then cooled to $20{ }^{\circ} \mathrm{C}$ and washed with $\mathrm{Et}_{2} \mathrm{O}(6 \times 15 \mathrm{~mL})$. The remainder was put under vacuum ( 0.5 Torr) for $2 \mathrm{~h}$ to afford the Bocprotected guanidinium salt $\mathbf{1 1}$. The Boc group was removed by adding $6 \mathrm{~N}$ aq. $\mathrm{HCl}(15 \mathrm{~mL})$ and refluxing for $4 \mathrm{~h}$ giving the tosylate 12 as a pale-yellow oil in $73 \%$ yield. $-{ }^{1} \mathrm{H}$ NMR $\left(300 \mathrm{MHz}, \mathrm{CDCl}_{3}\right): \delta=7.01(\mathrm{~d}, 2 \mathrm{H}, \mathrm{Ar}), 6.39(\mathrm{~d}, 2 \mathrm{H}$, Ar), $4.69(1 \mathrm{H}, \mathrm{NH}), 3.25\left(\mathrm{~m}, 2 \mathrm{H}, \mathrm{N}^{+}-\mathrm{CH}_{2}-\mathrm{CH}-\mathrm{NH}\right), 3.11$ $\left(\mathrm{m}, 2 \mathrm{H}, \mathrm{N}^{+}-\mathrm{CH}_{2}-\mathrm{CH}_{2}\right), 3.06\left(\mathrm{~m}, 1 \mathrm{H}, \mathrm{N}^{+}-\mathrm{CH}_{2}-\mathrm{CH}-\mathrm{NH}\right)$, $2.94\left(\mathrm{~m}, 2 \mathrm{H}, \mathrm{CH}_{2}-\mathrm{CH}_{2}-\mathrm{NH}\right), 2.55\left(\mathrm{~m}, 4 \mathrm{H}, \mathrm{N}-\mathrm{CH}_{2}-\mathrm{CH}_{3}\right)$, $2.33\left(\mathrm{~m}, 4 \mathrm{H}, \mathrm{N}-\mathrm{CH}_{2}-\mathrm{CH}_{2}-\mathrm{CH}_{3}\right), 2.52-2.47\left(\mathrm{~m}, 1 \mathrm{H}, \mathrm{CH}_{2}-\right.$ CH-NH), $2.27-2.19$ (m, 1H, $\left.\mathrm{CH}_{2}-\mathrm{CH}-\mathrm{NH}\right), 2.08-1.89$ (m, $\left.2 \mathrm{H}, \mathrm{CH}_{2}-\mathrm{CH}_{2}-\mathrm{NH}\right), 1.59$ (s, 3H, $\left.\mathrm{CH}_{3}-\mathrm{Ph}\right), 1.77-1.69$ (m, $\left.2 \mathrm{H}, \mathrm{N}^{+}-\mathrm{CH}_{2}-\mathrm{CH}_{2}\right), 1.52\left(\mathrm{~m}, 2 \mathrm{H}, \mathrm{N}^{+}-\left(\mathrm{CH}_{2}\right)_{2}-\mathrm{CH}_{2}\right), 1.44-$ $1.39\left(\mathrm{~m}, 4 \mathrm{H}, \mathrm{N}-\mathrm{CH}_{2}-\mathrm{CH}_{2}-\mathrm{CH}_{3}\right), 1.13\left(\mathrm{~m}, 2 \mathrm{H}, \mathrm{N}^{+}-\left(\mathrm{CH}_{2}\right)_{3}-\right.$ $\left.\mathrm{CH}_{2}\right), 1.10\left(\mathrm{~m}, 2 \mathrm{H}, \mathrm{N}^{+}-\left(\mathrm{CH}_{2}\right)_{4}-\mathrm{CH}_{2}-\mathrm{CH}_{3}\right), 1.01(\mathrm{t}, 6 \mathrm{H}, \mathrm{N}-$ $\left.\mathrm{CH}_{2}-\mathrm{CH}_{3}\right), 0.76\left(\mathrm{~m}, 3 \mathrm{H}, \mathrm{N}^{+}-\left(\mathrm{CH}_{2}\right)_{5}-\mathrm{CH}_{3}\right), 0.69(\mathrm{~m}, 6 \mathrm{H}, \mathrm{N}-$ $\left.\left(\mathrm{CH}_{2}\right)_{2}-\mathrm{CH}_{3}\right) .-{ }^{13} \mathrm{C} \mathrm{NMR}\left(75 \mathrm{MHz}, \mathrm{CDCl}_{3}\right): \delta=143.5$, 138.1, 127.6, 125.2, 114.3, 53.6, 50.1, 45.2, 45.0, 30.5, 28.8, 25.9, 21.8, 20.5, 20.1, 20.0, 13.3, 12.3, 12.2, 10.5. - HRMS: ((+)-ESI: $m / z=368.3866$ (calcd. 368.3868 for $\mathrm{C}_{22} \mathrm{H}_{48} \mathrm{~N}_{4}{ }^{+}$, $\left.[\mathrm{M}+\mathrm{H}]^{+}\right)$.

Typical experimental procedure for asymmetric direct aldol reaction (in excess of ketone as solvent) catalyzed by $\mathbf{4 a}$

A catalytic amount of catalyst $4 \mathbf{a}(58 \mathrm{mg}, 20 \mathrm{~mol}-\%)$ was added to a flask containing 4-nitrobenzaldehyde $(76 \mathrm{mg}$, $0.5 \mathrm{mmol})$ and cyclohexanone $(0.26 \mathrm{~mL}, 2.5 \mathrm{mmol})$ in a closed system under argon. The reaction mixture was stirred at $\mathrm{r}$. t. for $24 \mathrm{~h}$ and subsequently extracted with diethyl ether $(5 \times 10 \mathrm{~mL})$. The combined organic extracts were dried with anhydrous $\mathrm{MgSO}_{4}$ and the solvent was removed under re- duced pressure. The crude aldol product 15 was purified by silica-gel column chromatography $(n$-hexane/AcOEt $=4: 1)$ to afford the aldol product as a colorless solid $(118 \mathrm{mg}$, $94 \%$ yield).

\section{(S)-2-((R)-Hydroxy(4-nitrophenyl)methyl)cyclohexanone (15a)}

The diastereomeric anti:syn ratio was determined by ${ }^{1} \mathrm{H}$ NMR analysis of the crude product: $\delta=5.48(\mathrm{~d}, J=$ $1.8 \mathrm{~Hz}, 1 \mathrm{H}$, syn, minor), 4.89 (d, $J=8.8 \mathrm{~Hz}, 1 \mathrm{H}$, anti, major). Enantiomeric excess was determined by HPLC with a Chiralcel AD column ( $n$-hexane $/ i$-PrOH $90: 10,1.0 \mathrm{~mL} / \mathrm{min}, \lambda=$ $\left.254 \mathrm{~nm}, 25^{\circ} \mathrm{C}\right): t_{R}=26.3 \mathrm{~min}$ (minor) and $34.9 \mathrm{~min}$ (major). $-{ }^{1} \mathrm{H} \mathrm{NMR}\left(300 \mathrm{MHz}, \mathrm{CDCl}_{3}\right): \delta=8.20(\mathrm{~d}, J=8.6 \mathrm{~Hz}$, $2 \mathrm{H}), 7.50(\mathrm{~d}, J=8.7 \mathrm{~Hz}, 2 \mathrm{H}), 4.89(\mathrm{~d}, J=8.4 \mathrm{~Hz}, 1 \mathrm{H}), 4.07$ (s, 1H), $2.63-2.57(\mathrm{~m}, 1 \mathrm{H}), 2.49-2.32(\mathrm{~m}, 2 \mathrm{H}), 2.12-1.31$ $(\mathrm{m}, 6 \mathrm{H}) .-{ }^{13} \mathrm{C} \mathrm{NMR}\left(75 \mathrm{MHz}, \mathrm{CDCl}_{3}\right): \delta=214.8,148.4$, 147.6, 127.9, 123.5, 74.0, 57.2, 42.7, 30.8, 27.7, 24.7.

Typical experimental procedure for recycling catalyst $\mathbf{4 a}$ for asymmetric aldol reactions

The reaction mixture of the aldol reaction (vide supra) was extracted with diethyl ether $(3 \times 15 \mathrm{~mL})$. The combined organic layers were concentrated under vacuum, and after column chromatography the pure aldol product was obtained. The residual catalyst was concentrated under vacuum to remove residual ether and was reused for the next run.

\section{Acknowledgements}

Financial support by Bundesministerium für Bildung und Forschung (BMBF) is gratefully acknowledged. We thank Prof. Dr. Willi Kantlehner, Hochschule Aalen, for providing guanidines and guanidinium salts. We further acknowledge donation of chemicals from Saltigo $\mathrm{GmbH}$, Bayer Services $\mathrm{GmbH} \& \mathrm{Co}$. OHG, BASF AG and Sasol GmbH.
[1] M. J. Earle, K. R. Seddon, Pure Appl. Chem. 2000, 72, $1391-1398$.

[2] R. Sheldon, Chem. Commun. 2001, 2399-2407.

[3] N.S. Chowdari, D. B. Ramachary, C.I. I. I. Barbas, Synlett. 2003, $1906-1909$.

[4] P. Wasserscheid, W. Keim, Angew. Chem. 2000, 112, 3926-3945; Angew. Chem. Int. Ed. 2000, 39, $3773-$ 3789.

[5] J. Dupont, R. F. de Souza, P. A. Z. Suarez, Chem. Rev. 2002, 102, 3667-3691.

[6] C. C. Tzschucke, C. Markert, W. Bannwarth, S. Roller, A. Hebel, R. Haag, Angew. Chem. 2002, 114, 41364173; Angew. Chem. Int. Ed. 2002, 41, 3964-4000.

[7] R. A. Sheldon, R. M. Lau, M. J. Sorgedrager, F. van
Rantwijk, K. R. Seddon, Green Chem. 2002, 4, 147 151.

[8] J. Shah, H. Blumenthal, Z. Yacob, J. Liebscher, $A d v$. Synth. Cat. 2008, 350, $1267-1270$.

[9] R. Sebesta, I. Kmentova, S. Toma, Green Chem. 2008, 10, $484-496$.

[10] L. Y. Wu, Z. Y. Yan, Y.X. Xie, Y. N. Niu, Y. M. Liang, Tetrahedron: Asymmetry 2007, 18, 2086-2090.

[11] A. Zhu, T. Jiang, B. Han, J. Huang, J. Zhang, X. Ma, New J. Chem. 2006, 30, 736-740.

[12] W. Nan, W. Hai-Hong, J. Yong-Wen, Chin. J. Org. Chem. 2008, 28, 104-110.

[13] H. Kunkel, G. Maas, Eur. J. Org. Chem. 2007, $3746-$ 3757. 
[14] N. M. M. Mateus, L.C. Branco, N. M. T. Lourenco, C. A. M. Afonso, Green Chem. 2003, 5, 347-352.

[15] H. X. Gao, B. X. Han, J. C. Li, T. Jiang, Z. M. Liu, W.Z. Wu, Y. H. Chang, J. M. Zhang, Synth. Commun. 2004, 34, 3083-3089.

[16] M. G. Bogdanov, D. Petkova, S. Hristeva, I. Svinyarov, W. Kantlehner, Z. Naturforsch. 2010, 65b, 37-48.

[17] M. Tanaka, H. U. Siehl, T. Viefhaus, W. Frey, W. Kantlehner, Z. Naturforsch. 2009, 64b, $765-772$.

[18] J. Shah, J. Liebscher, Synthesis 2008, 917 - 920.

[19] S. S. Khan, J. Shah, J. Liebscher, Tetrahedron 2010, in print.

[20] Z. Yacob, J. Shah, J. Leistner, J. Liebscher, Synlett 2008, $2342-2344$.

[21] J. Shah, S.S. Khan, J. Liebscher, Synthesis 2009, $3975-3982$.

[22] S. S. Khan, S. Hanelt, J. Liebscher, Arkivoc 2009, xii, $193-208$.
[23] M. Lombardo, F. Pasi, S. Easwar, C. Trombini, $A d v$. Synth. Cat. 2007, 349, $2061-2065$.

[24] W. S. Miao, T. H. Chan, Adv. Synth. Cat. 2006, 348, $1711-1718$.

[25] M. Lombardo, S. Easwar, F. Pasi, C. Trombinia, $A d v$. Synth. Cat. 2009, 351, 276-282.

[26] D. E. Siyutkin, A.S. Kucherenko, M. I. Struchkova, S. G. Zlotin, Tetrahedron Lett. 2008, 49, 1212 1216.

[27] A.F. Spatola, M. K. Anwer, A. L. Rockwell, L.M. Gierasch, J. Am. Chem. Soc. 1986, 108, 825-831.

[28] N. Mase, Y. Nakai, N. Ohara, H. Yoda, K. Takabe, F. Tanaka, C. F. Barbas, J. Am. Chem. Soc. 2006, 128, $734-735$.

[29] Z. Tang, F. Jiang, L. T. Yu, X. Cui, L. Z. Gong, A. Q. Mi, Y.Z. Jiang, Y. D. Wu, J. Am. Chem. Soc. 2003, 125, $5262-5263$. 